\title{
CONTRIBUCIONES DE LA INSTITUCIÓN EDUCATIVA AL POSTCONFLICTO: HUMANIZARTE, UNA PROPUESTA PEDAGÓGICA PARA LA CONSTRUCCIÓN DE PAZ*
}

\author{
Carlos Valerio Echavarría Grajales** \\ Julián Bernal Ospina***: \\ Niky Alexander Murcia Suárez ${ }^{\text {*k.k.k }}$

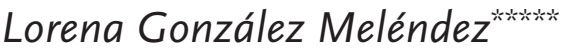 \\ Leyder Alonso Castro Beltrán
}

\footnotetext{
* doi: 10.11144/Javeriana.cao28-51.ciep. Este artículo es producto de la investigación “Programa de formación política, ética y ciudadana para la construcción de paz y convivencia escolar en las instituciones educativas del Distrito Lasallista de Bogotá; Humanizarte: propuesta pedagógica para la construcción de paz (derivada del Programa)" financiado por el Distrito Lasallista de Bogotá y Universidad de La Salle desde agosto de 2013 a diciembre de 2016. El artículo se recibió el 10/05/2015 y se aprobó el 15/11/2015. Sugerencia de citación: Echavarría G., C. V.; J. Bernal 0., N. A. Murcia S., L. González M. y L. A. Castro B. (2015). Contribuciones de la institución educativa al postconflicto: Humanizarte, una propuesta pedagógica para la construcción de paz. Cuadernos de Administración, 28 (51), 159-187. http://dx.doi.org/10.11144/Javeriana.cao28-51.ciep

** Doctor en Ciencias sociales, niñez y juventud de la Universidad de Manizales y CINDE, Manizales, Colombia, 2006. Docente de la Facultad de Ciencias de la Educación de la Universidad de La Salle, Bogotá, Colombia. Correo electrónico: cechavarria@unisalle.edu.co

*** Politólogo de la Universidad Autónoma de Manizales, Manizales, Colombia, 2015. Correo electrónico:julianbernalospina@gmail.com

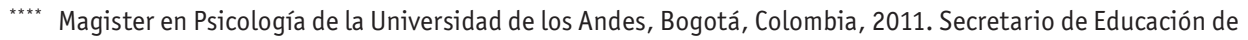
la Congregación de los Hermanos de las Escuelas Cristianas, Bogotá, Colombia.

Correo electrónico: nikyfsc@lasalle.org.co

${ }^{\star \star \star \star \star}$ Politóloga con énfasis en gobierno y relaciones internacionales de la Universidad Autónoma de Manizales, Manizales, Colombia, 2014.

Correo electrónico: loregom2@hotmail.com

${ }^{\star \star \star \star \star \star}$ Profesional en Filosofía y Letras de la Universidad De La Salle, Bogotá, Colombia, 2012. Investigador de la Congregación de Hermanos de las Escuelas Cristiana, Distrito de Bogotá, Bogotá, Colombia.

Correo electrónico: Investigaciónsed@lasalle.org.co
} 


\section{Contribuciones de la institución educativa al postconflicto: Humanizarte, una propuesta pedagógica para la construcción de}

paz

\section{Educative Institution}

Contributions to Post Conflict: Humanizarte, a pedagogical proposal for peace building

\section{Resumen}

Este artículo propone asumir la construcción de paz intrínsecamente relacionada con la constitución de un sujeto político y el fortalecimiento del ejercicio ciudadano de los actores. Las metodologías son cartografías sociales, entrevistas a profundidad y grupos focales. Un hallazgo es la necesidad de discutir la construcción de paz en relación con las virtudes públicas y el ejercicio ciudadano desde los derechos. Se concluye que la construcción de paz está relacionada con la transformación de imaginarios sociales, el desarrollo de capacidades y la implementación de pedagogías para la humanización, de lo cual se diseña la propuesta de formación política Humanizarte a ser implementada y validada.

Palabras claves: formación política, construcción de paz, instituciones educativas, propuesta pedagógica.

Clasificación JEL: I2, I26

\section{ABSTRACT}

This paper proposes to assume peace building as a process that is intrinsically related to the constitution of a political subject and the civic strengthening of its actors. The methodologies used are social cartographies, in-depth interviews and focus groups. One finding is the necessity to discuss peace building in relation with the public virtues and citizenship participation through citizens' rights. It concludes that peace building is related with the transformation of social imaginaries, the development of capabilities and the implementation of pedagogies for humanization, from which the political training proposal Humanizarte is designed for its application and validation.

Keywords: political training, peace building, educative institutions and pedagogical proposal.

JEL Classification: I2, I26

\section{Contribuições da instituição educativa para o pós-conflito: Humanizarte, uma proposta pedagógica para a construção da paz}

\section{Resumo}

Este artigo propõe assumir a construção da paz intrinsicamente relacionada com a constituição de um sujeito político e com o fortalecimento do exercício cidadão dos atores. As metodologias são cartografias sociais, entrevistas a profundidade e grupos focais. Uma constatação é a necessidade de discutir a construção da paz fazendo relação com as virtudes públicas e com o exercício cidadão a partir dos seus direitos. Conclui-se que a construção da paz está vinculada à transformação de imaginários sociais, ao desenvolvimento de capacidades e à implantação de pedagogias para a humanização. A partir disso, desenha-se a proposta de formação política Humanizarte, a ser implantada e validada.

Palavras-chave: formação política, construção da paz, instituições educativas, proposta pedagógica.

Classificação JEL: I2, I26 


\section{Introducción}

En este artículo se expone -a manera de hipótesis- que responder a los desafíos de la construcción de paz en Colombia implica el diseño, la implementación y la evaluación de propuestas educativas que afecten los imaginarios sociales sobre paz y convivencia, el desarrollo de capacidades éticas y política de los miembros de las comunidades educativas, y la reflexión de las prácticas pedagógicas de quienes están al frente de la enseñanza y de las prácticas de gobernabilidad de quienes administran y gestionan el quehacer educativo.

Con el propósito de brindar argumentos que contribuyan a sustentar la hipótesis planteada, este escrito se estructuró en cuatro partes: en la primera, se presentan los antecedentes teóricos e investigativos que fundamentan la pregunta por los imaginarios sociales de paz y convivencia en la escuela ${ }^{1}$. Se determina en qué consiste la función pública de la escuela y se hace una descripción breve del área problemática de la investigación.

En la segunda parte se expone la metodología utilizada en la primera fase del Programa de investigación, la cual consistió en un estudio cualitativo desde una perspectiva hermenéutica comprensiva. Se hace una descripción de los fundamentos epistemológicos y metodológicos del estudio, se presentan las técnicas e instrumentos utilizados para dar respuesta a la pregunta central del estudio y se hace una descripción del proceso metodológico.

En la tercera parte se describen los principales hallazgos de la investigación sobre imaginarios sociales de convivencia y paz. Inmediatamente, se expone la propuesta pedagógica Humanizarte. Esto, con la finalidad de indicarle al lector que esta iniciativa pedagógica hace parte de los hallazgos del Programa, puesto que fue diseñada para enfrentar los problemas de convivencia y paz que las instituciones educativas del Distrito Lasallista de Bogotá (DLB) identificaron en el proceso de exploración por los imaginarios sociales de convivencia y paz.

Finalmente, en la cuarta parte del artículo se propone una discusión y se derivan algunas conclusiones relacionadas con los aportes de las instituciones educativas a la construcción de paz en tiempos de postconflicto.

1 Esta revisión de antecedentes investigativos (empíricos y teóricos) relacionados con la construcción de paz (peace building) y la educación para la paz (peace education), fue desarrollada en las bases de datos de ProQuest, EbscoHost y Scopus. 


\section{Fundamentos teóricos e investigativos}

La construcción de paz en el postconflicto no solo es un propósito formativo que se logra cuando las instituciones educativas trabajan por una sana convivencia y resolución pacífica de los conflictos; es también una conquista social y política que involucra a diversos sectores -económicos, políticos, estatales y educativos- a la vez que exige un análisis histórico más detallado de las condiciones que han favorecido el conflicto armado y la violencia, y que han desestimado el buen vivir, la justicia y la equidad.

La construcción de paz en el contexto colombiano ha cobrado mayor interés por parte de diversos actores (Estado, sociedad civil, comunidad internacional, actores armados), todos ellos interesados en hallar una resolución política al conflicto armado. Desde esta perspectiva, "construir paz requiere enfrentar el acumulado de traumas y daños (individuales tanto como colectivos, directos como indirectos) que se manifiestan en síntomas de indiferencia, escepticismo, depresión, desconfianza, temor, dolor, rabia y odio. Para enfrentar este trauma, hace falta construir memoria histórica, reconstruir el tejido social y las confianzas cotidianas rotas, y promover la restitución y las reparaciones transformadoras individuales y colectivas" (Bouvier, 2014, p. 13).

Alcanzar logros de construcción de paz está directamente vinculado con el fortalecimiento político de la sociedad civil para organizarse y contribuir a posicionar la paz como una prioridad pública nacional y de interés para toda la ciudadanía (Isacson y Rojas, 2014; PNUD, 2003). También, este fin social se consigue con el involucramiento de todos los sectores: el educativo, para el desarrollo de actitudes, creencias, valores y comportamientos (Chaux y Velázquez, 2014; Niermann, 2012, citado por Jäger, 2014); el eclesial, en la identificación del dolor de las víctimas y su asistencia hasta que el tejido social se repare (Henao, 2014); el empresarial, en proponer estrategias para asumir los costos de la construcción de paz, "invirtiendo tiempo y recursos en el fortalecimiento de instituciones, en la organización de las comunidades, en el mejoramiento de la gobernabilidad local, en el apoyo a programas de educación, empleo y resolución pacífica de conflictos" (Rettberg, 2014, p. 250). A su vez, lograr la paz se alcanza en el reconocimiento del trabajo cooperado de movimientos tanto de género como étnicos (Rojas, 2014; Wirpsa, Rothschild y Garzón, 2014). Y, por último, en la identificación del potencial que tienen las iniciativas locales y regionales de paz para promover la participación política y la organización social y comunitaria requerida en la constitución de territorios de paz (Mitchell y Ramírez, 2014; Moncayo, 2014; Roldán, 2014; Esquivia y Gerlach, 2014; Ramírez, 2014). 
En cuanto a la relación construcción de paz vs. educación para la paz, según las investigaciones revisadas, es posible identificar tres hipótesis de trabajo, a saber: la primera de ellas, se refiere a la necesidad de entender que la construcción de paz no es ausencia del conflicto. Por el contrario, se asume que el conflicto es consustancial a las relaciones entre los seres humanos y, por tanto, es un elemento fundamental para repensar la paz y las variadas formas en que los seres humanos lo resuelven bajo estrategias no violentas, como la creación de ambientes educativos de sana convivencia y de reconocimiento de la diversidad y la diferencia. Para ello se propone la relación entre construcción de paz y memoria histórica, mediante la propuesta de museos como escenarios educativos de la paz (Gachanga y Mutisya, 2015; Tamashiro y Furnari, 2015). También, este enfoque pedagógico de convivencia y paz es un elemento trascendental para estudiar con detenimiento las estructuras sociales y culturales que originan principalmente los conflictos $y$, de esa manera, evitar cualquier emergencia (o reemergencia) de la violencia (Gur-Ze'ev, 2010; Adetoro, 2015; Galtung, 1985; Harber y Sakade, 2009; Power, 2014; Page, 2014; Salomon y Cairns, 2010; Jäger, 2014).

La segunda hipótesis es la relación directa entre la construcción de paz y el fortalecimiento de una idea de Estado. A la luz de esta relación, este se comprende como una institución que debe crear y garantizar las condiciones para la paz. Según el artículo 22 de la Constitución Política de Colombia, "La paz es un derecho y un deber de obligatorio cumplimiento". Se comprende como un deber en tanto involucra a toda la ciudadanía colombiana desde su aspecto más formal, relacionado con el Estado y sus instituciones, hasta el más emergente, relacionado con lo social y las demandas de la sociedad civil, que con el paso del tiempo se vuelve cada vez más apremiante, e implica que los distintos sectores y los ciudadanos deban crear las condiciones para garantizar una paz sostenible. Planteamientos similares se hallan en Webel (2007), Page (2014), Galtung (1990) y Arévalo (2014).

La tercera hipótesis de trabajo establece una relación entre la construcción de paz y la transformación de una cultura de violencia hacia la consolidación de una cultura de paz. Jäger (2014) citando a Nipkow (2007) plantea que la educación para la paz tiene la difícil tarea de transformar los hábitos por medio de los cuales se naturalizan la ausencia de paz y el uso de la violencia y la guerra como medios políticos de resolución de las situaciones conflictivas. Las oportunidades para incrementar la paz, según Jäger (2014) citando a Wulf (2007), dependen de que las personas aprendan a reconocer su imaginación relevante de paz. 
Desde esta tercera hipótesis, la educación para la paz contribuye a promover valores, actitudes, conocimientos, habilidades, creencias, atributos y prácticas sustentados en valores de paz, de no discriminación y de dignidad humana. En este sentido, la educación para la paz posibilita un comportamiento pacífico y el hallazgo de soluciones no violentas a conflictos surgidos en las relaciones sociales y en contextos violentos y de guerra (Harber y Sakade, 2009; Power, 2014; Adetoro, 2015; Page, 2014; Salomon y Cairns, 2010; Jäger, 2014).

Por consiguiente, la educación y la escuela tienen el reto de construir paz en escenarios violentos. Para tales efectos, un aspecto que reluce en la medida en que se pretende inquirir por una educación para la paz son las metodologías que promueven diálogos, transmiten conocimientos y brindan espacios para la paz (Adetoro, 2015; Gachanga y Mutisya, 2015; Jäger, 2014; Tabulawa, 2013; Thapa, Cohen, Guffey y Higgins- D’Alessandro, 2013; Harber y Sakade, 2009). Según Adetoro (2015), tanto los métodos convencionales basados en la transmisión de contenidos como los métodos alternativos basados en la enseñanza hacia el estudiante (denominados Learning Together y Constructive Controversy) son necesarios para promover una adquisición de conocimientos y habilidades para la solución pacífica de los conflictos.

Por su parte, Jäger (2014) establece que la educación para la paz se preocupa por estrategias integradas y holísticas guiadas por el concepto de paz, a través de las cuales se buscan promover distintas formas de diálogo constructivo para la paz y la transformación de conflictos, lo cual significa ayudar a formar tanto en habilidades grupales como individuales. Harber y Sakade (2009) concluyen que la educación para la paz y la educación formal o normal son diferentes tanto en las intencionalidades como en la práctica misma. Un ejemplo de esta tensión evidenciada es la forma en que se concibe el profesor: como un facilitador que potencia la autonomía y la responsabilidad de sus estudiantes (caso de la educación para la paz) o como un controlador que pretende disciplinarlos y ordenarlos (caso de la educación formal o normal).

De otro lado, Tabulawa (2013) relativiza la idea según la cual un método pedagógico es superior o inferior de otro. Establece que las posibles fallas en los métodos pedagógicos son consecuencia del contexto social o de lo que denomina como "la estructura social envolvente". En ese sentido, el análisis de la práctica pedagógica debe tener en cuenta la naturaleza social de la pedagogía y los diversos factores que atraviesan la realidad social. Salomon (2004) -en Jäger (2014) - plantea la necesidad de una contextualización dependiendo de la tipología de conflicto. De ahí que se haga énfasis en que la educación 
para la paz ha estado ligada a las problemáticas que surgen de los contextos políticos (conflictos sociales, conflictos armados, guerras) particulares de los países. De esa manera, las temáticas que se relacionan dependen en gran medida del grado de incidencia que tengan los valores e ideologías que en tal o cual momento se promuevan, de quiénes se han constituido como los movimientos sociales que han desarrollado o promovido la agenda pública, y de las representaciones e imaginarios sociales que configuran las interacciones entre los individuos.

En cuanto a la función política de la escuela en tiempos del postconflicto, su rol está atravesado por categorías sociopolíticas que se enmarcan más allá del contexto educativo como tal. Grau (2013) afirma que para no correr el riesgo de revictimizar y perpetrar la exclusión estructural hacia las mujeres o hacia quienes históricamente han sido marginalizados, es perentorio empoderar a estas poblaciones y exponer las exclusiones y demás injusticias sociales de que han sido objeto. Este propósito de la construcción de paz guarda total coherencia con lo que plantea Fernández (2014), quien propone que la formación política no solo debe enfatizar en cómo se aprenden a identificar los síntomas de las problemáticas sociales y sus posibles causas, sino también en cómo involucrarse en el desarrollo de propuestas que permitan superar los efectos de la exclusión de grandes sectores de la población.

Con base en estas afirmaciones podría inferirse que una de las funciones políticas de la institución educativa es generar transformaciones culturales y sociales favorables a la consolidación de prácticas y valores de paz, representaciones de igualdad y justicia, inclusión, buen vivir y democracia. Para esta finalidad educativa, una posible estrategia es que las niñas, los niños y los jóvenes aprendan sobre la historia del país, identifiquen con precisión las implicaciones que ha acarreado la violencia estructural en las condiciones de vida de la ciudadanía y discutan sobre los desafíos sociales, económicos, culturales y políticos de lo que significa aprender a vivir juntos en una nación que se denomina a sí misma como un Estado social de derecho.

Es así como la escuela no solo tiene una labor comprensiva de los problemas que enfrentan sus miembros, sino también una labor transformadora de la cultura y de la sociedad. Algunos expertos han identificado claramente que la educación es una herramienta potente para estos efectos transformadores y sin la cual no se podrían dar los desarrollos en la cultura, la ciencia y la tecnología de un país (Echavarría y Vasco, 2013; Sarmiento, 2011; Orozco, 2012; Comisión de Sabios, 1996). 
Nussbaum $(2011,2012)$, por su parte, desde un enfoque psicológico y filosófico, establece que la escuela contribuye a desarrollar las capacidades de los alumnos para que aprendan a ver el mundo desde la perspectiva del otro y para que comprendan lo fundamental que resultan la cooperación y la reciprocidad para la construcción de paz.

Desde esta perspectiva, la escuela tiene el deber de reflexionar con especial cuidado cómo se propician y se fortalecen las interacciones sociales en función del buen vivir y el florecimiento humano. Complementariamente, debe crear espacios para discutir y proponer estrategias conducentes al establecimiento de relaciones democráticas e incluyentes y para formalizar procedimientos profundamente democráticos, fundamentados en criterios de justicia, cuidado y reconocimiento, y comprometidos con la promoción de derechos (Echavarría, Murcia y Castro, 2014).

Dadas estas menciones de la función política de la escuela, la construcción de paz y convivencia en las instituciones educativas constituye una intención educativa no solo posible y ejecutable sino también de carácter trascendental para nuestro país. En ese sentido, se configura en este artículo la noción según la cual la escuela, a través de la transformación de prácticas, discursos y modos de enseñanza de los maestros con la intención de construir escenarios de paz, contribuye al desarrollo de actitudes, valores, conocimientos y prácticas políticas y ciudadanas. Lo anterior, con miras a fortalecer la democracia, el ejercicio político-ciudadano y las prácticas de concertación de conflictos, a partir de reflexiones y discusiones acerca de los correlatos morales que le subyacen a toda práctica política.

Una exploración general de las investigaciones empíricas en el marco de la función política de la escuela presenta un panorama favorable para brindar distintas luces de articulación de esta tesis, pero a su vez permiten hallar un locus teórico de análisis a partir de la reflexión de la transformación de las prácticas pedagógicas². En la literatura revisada, es posible inferir que las diversas iniciativas de construcción de paz en la escuela enfatizan primordialmente en aspectos relacionados con la configuración de subjetividades e imaginarios políticos, el desarrollo de capacidades (actitudes, valores y conocimientos) políticas, la comprensión del conflicto y la agresión escolar (Gómez, 2013; Chaux, 2012; Díaz, 2011; Alvarado, Botero y 0spina, 2010; Torrente y Kanayet, 2006; Mestizo, 2000). Proponen como apuesta política fundamental garantizarles a las niñas, los niños y los

2 Esta parte de antecedentes fue tomada de Echavarría, Murcia y Castro (2014). 
adolescentes el reconocimiento y la legitimidad en su palabra, de manera tal que sea tenida en cuenta como parte fundamental de la toma de decisiones, el desarrollo de su propio potencial humano y la construcción de una confianza básica conjunta (Save the Children, 2008; Carter y Shipler, 2005). Algunas estrategias están articuladas al sentido de la deliberación normativa, la discusión sobre problemáticas que afectan la interacción entre los seres humanos y entre estos y el medio ambiente y la relación entre memoria y construcción de paz (Tanigawa, 2015; Watanabe, 2015; Lewis y Khatari, 2015; Tamashiro y Furnari, 2015; Verhagen, 2014; Naoufal, 2014).

Desde un enfoque disciplinar acerca de la convivencia y la paz en la escuela, se indaga por la información sobre lo que se debe conocer y hacer por los niños, las niñas y los adolescentes, describiendo los factores, emociones, condiciones, actitudes y conductas agresivas que configuran un ambiente escolar violento como justificación de cualquier intervención pedagógica (López de Mesa, Carvajal, Soto y Urrea, 2013; Chaux, 2012; Alvarado, 2010).

Otro grupo de investigaciones se enfoca, primordialmente, en las prácticas pedagógicas y en las iniciativas que maestras y maestros plantean para promocionar la inclusión social y crear ambientes de aprendizaje alternativos en los que niñas, niños y adolescentes diversos en su cognición, en orientación sexual, en su género y en su etnia puedan recibir una educación de calidad sin ser discriminados (Bajaj, 2015; Ross, 2015; Gómez, 2013; Díaz, 2011; Torrente y Kanayet, 2006; Galvis, Moya, Garcés, Morales y Galeano, 2006).

En cuanto a las limitaciones en la consolidación de una función política con intención de construir paz, unas investigaciones ilustran restricciones de la educación en cuanto ésta no atiende de manera particular los desafíos relativos a la inclusión de niñas, niños y adolescentes con discapacidad, vulnerados o con necesidades educativas especiales (Pinto, Torres, González y Caicedo, 2012; Galvis et al., 2006) o pertenecientes a comunidades afro (Rojas et al., 2010). Otras investigaciones hacen énfasis en caracterizar el tema de la violencia escolar desde la identificación de factores asociados con la agresión tales como el clima escolar, las actitudes agresivas y algunas formas de relación (López de Mesa et al., 2013), aunque buena parte de las investigaciones sean más descriptivas que analíticas de las categorías de la agresión y la violencia escolar (Ramírez y Arcila, 2013). De manera complementaria, estas investigaciones presentan un panorama amplio en cuanto a metodologías participativas para el diseño de propuestas de formación; realizan análisis multivariados de comprensión de la agresión y la violencia al interior de la escuela; y proponen articulaciones de la construcción de paz con alternativas pedagógicas de transformación de valores, actitudes y formas de negociación de los conflictos. 
De los estudios revisados se puede concluir que la mayoría de indagaciones no trascienden las aulas de clase y más que configurarse como una propuesta institucional, quedan circunscritas a la buena voluntad de los maestros y las maestras, mientras que algunas investigaciones se ubican en espacios de educación para la paz que no necesariamente son la escuela (Gachanga y Mutisya, 2015; Tamashiro y Furnari, 2015; Tanigawa, 2015; Watanabe, 2015; Lewis y Khatari, 2015; Bajaj, 2015). Un elemento adicional que caracteriza la mayoría de estudios, además de enfocarse en las prácticas pedagógicas, es la relación que se entabla entre el derecho y la pedagogía para argumentar la importancia que tiene para la niñez el que sean promovidos sus derechos desde la escuela. No obstante, el trabajo que se realiza bajo esta orientación queda más como un asunto normativo externo y no como un aspecto central de las interacciones, procesos y procedimientos que regulan la convivencia.

Finalmente, desde estas investigaciones se reconoce la importancia de diagnosticar la convivencia y la paz desde los asuntos patológicos, el acrecentamiento de la violencia escolar y bullying, los ambientes claramente marcados por conductas de agresividad, indisciplina y falta de control de las emociones, razón por la cual la toma de decisiones por parte de niñas, niños y adolescentes se torna problemática porque no hay conciencia de las consecuencias que acarrean, por ejemplo, las agresiones.

En términos generales, las investigaciones revisadas permiten inferir un camino de indagación y de intervención pedagógica que ilustra cómo la construcción de subjetividades políticas, el desarrollo de competencias ciudadanas y la educación intercultural contribuyen a la mitigación de la violencia escolar y de las agresiones que pueden darse entre estudiantes. También, reconocen la relevancia del derecho como criterio fundamental de regulación de las interacciones entre maestros y estudiantes.

No obstante, la mayoría de discusiones y aportes de estos estudios no presentan con detenimiento y a profundidad una discusión sobre los asuntos éticos, morales y políticos que están implicados con el ejercicio de la ciudadanía, la vivencia de los derechos y la configuración de escenarios para la paz y la convivencia desde la escuela.

Aunque se reconoce la importancia de transformar los valores, las actitudes, los imaginarios y las conductas de niñas, niños y adolescentes esta intencionalidad es absolutamente incompleta si no se generan también transformaciones en las prácticas, los discursos y los modos de enseñanza de maestras y maestros, así como también en la manera como está concebida y estructurada la institución educativa. 
Si bien en todo diagnóstico educativo es significativo identificar los factores potenciadores que conllevan al desarrollo de capacidades, posibilidades, condiciones institucionales y pedagógicas para la construcción de paz, también es importante reconocer y someter a discusión aquellas prácticas pedagógicas -configuradas en la cotidianidad del maestro y de la maestra- que proveen un saber absolutamente importante para sostener la paz y la convivencia. A su vez, se hace necesario analizar y promover los escenarios de construcción de garantía de los derechos fundamentales y de práctica de oportunidades para refrendar y transformar, cuando sea necesario, los acuerdos mínimos éticos, morales y normativos que regulan las interacciones en las instituciones educativas.

Finalmente, las investigaciones revisadas documentan los diversos desafíos que enfrentan la construcción para la paz y la educación para la paz. De manera particular, las investigaciones exponen los retos que debe afrontar la escuela, de lo cual se deriva una pregunta por el cómo reestructurarse para confrontar los impactos de la violencia, máxime cuando paulatinamente la cultura de masas debilita su monopolio cultural y deja de ser un orden regular para convertirse en una burocracia profesional (Dubet, 2004), y cuando se espera que los maestros promuevan actitudes más democráticas en estructuras institucionales jerárquicas.

En motivo de lo expuesto, con la intención investigativa de ahondar en la función política de la escuela y en su rol para la construcción de paz y convivencia escolar, el Programa de formación política, ética y ciudadana para la construcción de paz y convivencia escolar (DLB y Universidad de La Salle) planteó, en su primer año, la pregunta de investigación con miras a la identificación de las variadas dinámicas que estudiantes, maestros, directivos y personal de apoyo articulaban con respecto a la construcción de paz y convivencia (Echavarría et al., 2014). Para ello, se indagaron las prácticas de interacción, las representaciones simbólicas y los criterios éticos y morales de enjuiciamiento de las prácticas de construcción de paz y convivencia.

\section{Metodología}

Dar cuenta por los imaginarios sociales de convivencia y paz implicó un diseño metodológico descriptivo-hermenéutico, puesto que se buscó fundamentalmente comprender las variadas discusiones que 80 miembros de las comunidades educativas del DLB tenían con respecto a la convivencia y la paz. 
Para facilitar el trabajo de campo en grupos colegiados de estudiantes, maestros, padres de familia y directivos, como metodología se utilizó el taller pedagógico. En total se realizaron tres talleres por cada una de las 14 instituciones educativas que participaron de la investigación. Las técnicas e instrumentos que se seleccionaron fueron la realización de cartografías sociales y políticas, las entrevistas a profundidad y los grupos de discusión ${ }^{3}$.

Como puede observarse en la Figura 1, la primera fase del Programa de formación política, ética y ciudadana para la construcción de paz y convivencia en instituciones educativas del DLB, pretendió propiciar escenarios para la reflexión de la acción y de la práctica en los que los implicados, a través de sus intervenciones, análisis, comprensiones y perspectivas pudieran participar activamente en la concreción de la transformación de prácticas (construcción conjunta de alternativas de cambio).

\section{Hallazgos}

Un elemento fundamental de construcción de paz y convivencia en la escuela, según se reporta en los resultado de la primera fase de este programa (Echavarría et al., 2014), está directamente relacionado con la percepción positiva que niñas, niños y adolescente tienen de la institución y de cómo están estructuradas las relaciones de poder, puesto que a partir de esta percepción se legitiman ciertas prácticas, que si bien aún están dentro de las lógicas jerárquicas del poder, también pueden ser consideradas como formas posibles y necesarias para sostener una intención formativa hacia el florecimiento humano. Las valoraciones hechas a las relaciones que se establecen al interior de las instituciones dependen de las circunstancias cotidianas que enfrentan quienes interactúan ${ }^{4}$. Por ejemplo, se valora de manera distinta a un coordinador, profesor o padre de familia cuando determina colegiadamente los límites de la interacción, cuando explicita y comunica

3 La cartografía social y política permite, por medio de las representaciones de espacios y territorios definidos en los que se habita cotidianamente, reconstruir y representar simbólicamente el saber, reactualizar la memoria colectiva y reconocer los niveles de apropiación de los actores con respecto a los territorios. Los participantes dibujan, tanto individual como colectivamente, los territorios, los actores y las interacciones entre ellos, lo cual les posibilita conocer la realidad para actuar en ella, construir y legitimar conocimientos, reconstruir y reconfigurar experiencias y elaborar propuestas de transformación, todo esto en un diálogo constante de saberes. De otro lado, las entrevistas fueron diseñadas de manera semiestructurada para la profundización de las problemáticas asociadas con la paz y la convivencia, la validación de los elementos (criterio ético e investigativo) y para la objetivación intersubjetiva del conocimiento (criterio científico). En cuanto a los grupos de discusión, fueron diseñados para dialogar las causas y las consecuencias de las problemáticas en convivencia y paz de las instituciones educativas del Distrito Lasallista de Bogotá.

4 Esta descripción de hallazgos fue tomada de Echavarría et al. (2014). 


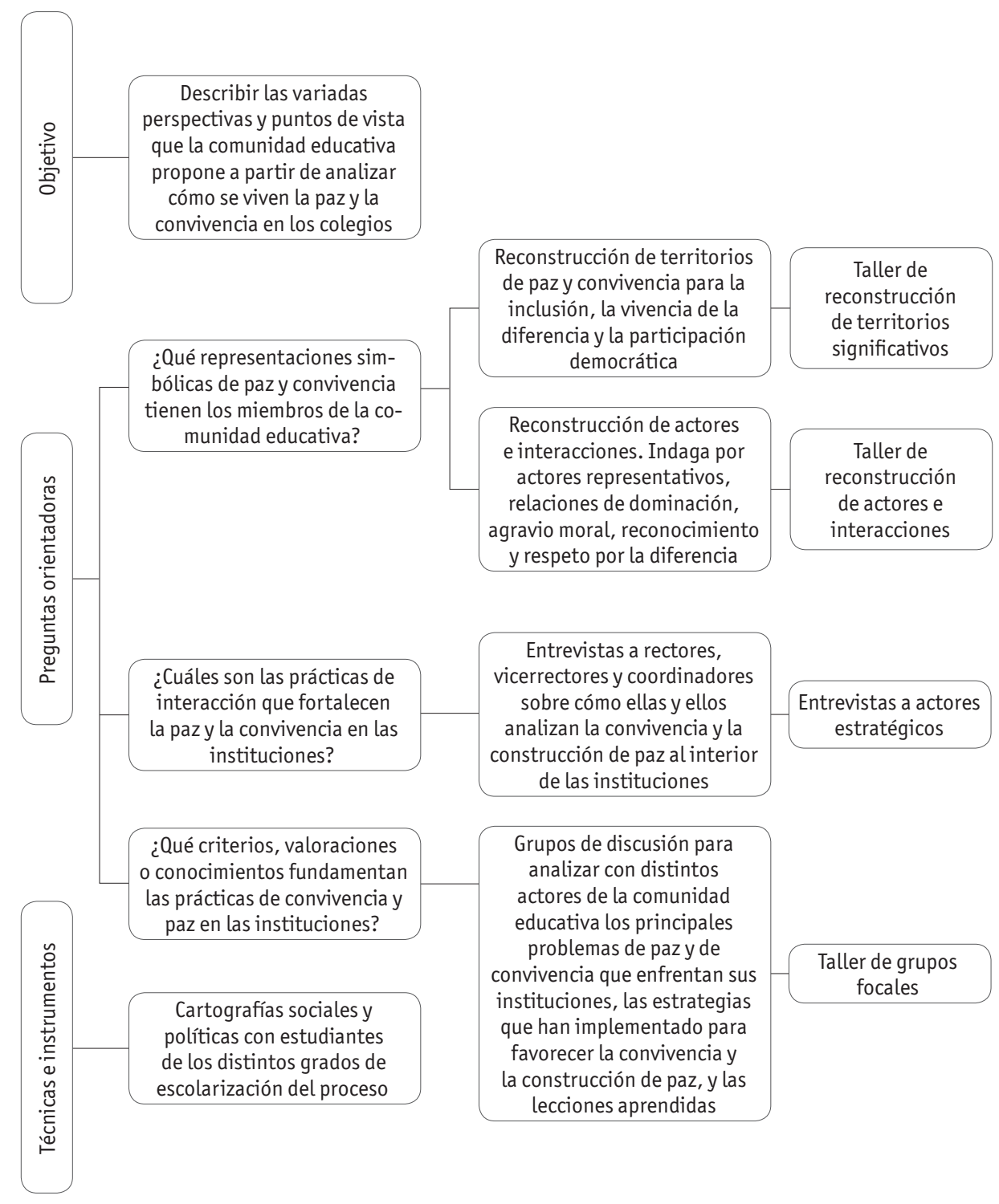

\section{Figura 1. Proceso metodológico}

Fuente: elaboración propia.

oportunamente los criterios fundamentales de la sanción o no de un estudiante, cuando simplemente se impone el orden sin mayores explicaciones, se exige una norma, se impone un castigo de manera ligera, arbitraria y sin la posibilidad de diálogo, o cuando 
el proceso de regulación se ha mecanizado tanto que no reporta aprendizajes sino un vaciamiento del sentido normativo de la regla. Como lo dijo un joven: "se pierde la posibilidad de aprender".

Un testimonio que apoya e ilustra estas inferencias:

Las interacciones con algunos Hermanos, coordinadores, profesores y algunos compañeros son relaciones de comunicación y fluidas porque la van bien, todos los días hablan, se entienden, no se ven tantos problemas y hay una buena comunicación por condiciones de amistad, respeto, amabilidad, buen humor, enseñanza coherente al reprender, confianza, manifestaciones de cariño, comprensión y tranquilidad al escuchar (NSG-I1) $)^{5}$.

Por el contrario, romper con la expectativa social promocionada en el proyecto educativo, tiene como consecuencias fracturas en las relaciones y generación de violencia:

Irrespeto de algunos profesores, no saben corregir (...) no hay libre expresión, no escuchan (...) no se les puede decir nada, creen que solo ellos tienen la razón. Son muy agresivos e imponentes porque sus órdenes son la última palabra (...) cuando deciden algo y no tienen en cuenta la opinión de nosotros (NSG_I1).

La claridad con que la comunidad educativa perciba positivamente el proyecto educativo institucional posiciona con mayor relevancia la labor que cumplen los colegios en las comunidades.

En la entrevista a profundidad que se realizó con un grupo de estudiantes, maestros, directivos y padres de familia, de uno de los colegios del DLB, estos coincidieron en afirmar que su colegio es un lugar de cuidado, de protección y respeto. Alli “se vive como en una burbuja", manifestó un joven. Una madre de familia plantea categóricamente que desde que los Hermanos de La Salle tomaron el colegio se ha ido cambiando paulatinamente la cultura y las formas de relacionarse con la educación de sus hijos. Antes-dice la madre"uno venía al colegio casi sin arreglarse, pero ahora todos venimos bien presentados y con buena disposición a escuchar sobre los procesos de nuestros hijos".

5 Al final de cada testimonio citado se especifica una sigla que corresponde a la codificación de la información que realizaron los investigadores para diferenciar las fuentes de origen: entrevista, grupo de discusión. 
De esta manera, la existencia de un proyecto educativo socializado, concertado y delimitado en los principios básicos que orienten el quehacer educativo y los modos legítimos de interacción, representa un buen punto de partida desde el cual regular las interacciones y derivar los criterios y principios de la acción pedagógica. Quizá, este elemento adquiere mayor relevancia cuando se explicitan los valores, principios y normas que producen mayor vínculo social. También cuando las prácticas cotidianas -rutinas, hábitos, formas de interacción- procuran estar en coherencia con aquellos principios que se pretenden alcanzar.

Un segundo elemento que contribuye a la construcción de paz y convivencia está en explicitar de qué manera el acto educativo está orientado a la transformación social y cultural de los estudiantes. Es decir de qué manera el paso por el colegio brinda oportunidades de ascenso social, lucro, proyectos de vida alternativos y fortalecimiento de la democracia. En este sentido, un directivo de una de las instituciones del DLB afirma:

La transformación comienza cuando se da la dignificación de las personas. [...] Así nosotros los adultos, le apuntamos a un tema bien importante, por su puesto direccionado desde los Hermanos, la dignificación de la personas. Yo creo que cuando nosotros como docentes, como coordinador, señora de servicio generales, como el portero, entendemos el concepto, el de persona primero para poder dignificarla, yo creo que es ahí donde comienzan esas transformaciones. [...] claro que si hay problemas, pero comparado con el contexto, se asimilaría más a faltas leves que graves. Por lo menos, hemos ganado en la dignificación de las personas, por ello, se entiende que este colegio es un territorio de paz (Tomado JLL-B0.V2).

En la narrativa se concibe la construcción de paz desde la dignificación de las personas; lo cual permite inferir que la paz y la convivencia no solo es un resultado que se alcanza cuando vivimos adecuadamente bajo criterios normativos, sino también cuando sentimos y percibimos que nos estamos dignificando. La dignificación de los humanos (capacidad y posibilidad de hacer y ser digno por sí mismo), categóricamente, se afirma en este relato, contribuye a la transformación de sí mismo y del entorno social y cultural adverso a los deseos de un proyecto de vida.

Dignificar al otro, como requisito central de construcción de paz y convivencia está, adicionalmente relacionado con entender su situación, con percibir qué tipo de adversidad enfrenta y con considerar cuál es el tipo de apoyo y solidaridad que requiere. Como lo ilustra una maestra: 
La convivencia fue fuertemente trabajada en equipo desde la comprensión de los problemas que enfrentaban los estudiantes. Ese era el punto: el punto lasallista, punto de reflexión, el punto que nos tocaba a uno como ser humano, muchas problemáticas que a uno no le había tocado vivir. [...] Cada uno de los profesores que les ha tocado pasar por acá nos metimos en los zapatos de las familias y en los zapatos de los chicos y, lo que dice Ronald es cierto, vivimos con ellos, estamos con ellos, nos sentimos una familia, lo que les afecta a ellos, nos afecta a nosotros, lo que uno puede decir que pedagógicamente es utópico, no. Si existe el cambio (Tomado JSLL-Bo.V2).

En el relato de esta maestra se encuentran varios elementos que están relacionados con la dignificación humana. El primero de ellos, está relacionado con percibir la adversidad que enfrentan los otros, valorar su condición y tomar la decisión de hacer algo. El segundo, construir con el otro una condición no adversa, brindando posibles alternativas de cambio. Y el tercero, creer en la pedagogía como una práctica realmente transformadora de las condiciones de adversidad que pueden enfrentar, sin proponérselo, los estudiantes que cotidianamente están en las instituciones educativas del DLB.

Un tercer elemento de construcción de paz y convivencia está en promover una pedagogía transformadora que reconozca en los estudiantes su potencial para ser dignos por sí mismos y para proponer alternativas de vida que conduzcan a su propio florecimiento. Una maestra lo expresa así:

La experiencia al interior de nosotros como docentes y de nuestras familias personalmente, a veces, uno como que está inconforme, está en confort, está en lo chévere, digamos, en que ninguna situación adversa a la vida de uno lo toca. Cuando uno llega al colegio y, digamos que uno se da cuenta de que si es cierto que existen problemáticas diferentes a la vida de uno y, uno dice esto no me ha pasado, cómo lo puedo solucionar [...] A nivel de pedagogía nosotros decimos la pedagogía y la educación es utópica, de que los estudiantes lleguen a cambiar es utópico, eso no es cierto, es un vacío. Cuando uno llega a este colegio se da cuenta de que sí es cierto cambiar, de que sí es cierto que la educación que nosotros brindamos es buena para ellos, es darle una proyección de vida (Tomado JLL-Bo-V2).

En este relato, la maestra reconoce la importancia de su labor, específicamente en el impacto que puede llegar a tener sus enseñanzas en los proyectos de vida de sus estu- 
diantes. También brinda una excelente justificación de la importancia de la educación y de la pedagogía en la transformación social y cultural de los miembros de una comunidad educativa.

\section{Propuesta pedagógica de formación política, ética y ciudadana para la construcción de paz: Humanizarte}

Como parte fundamental de los hallazgos de esta investigación, se diseña la propuesta pedagógica de formación política, ética y ciudadana para la construcción de paz, Humanizarte. Esta iniciativa está sustentada en tres ideas básicas articuladas desde las siguientes hipótesis: (1) todo proceso de construcción de paz está intrínsecamente relacionado con el desarrollo de capacidades políticas, éticas y ciudadanas; (2) todo proceso de construcción de paz está orientado a la transformación de imaginarios sociales; (3) todo proceso de construcción de paz está direccionado desde prácticas pedagógica de humanización y cultivo de la humanidad.

El desarrollo de capacidades políticas, ética y ciudadanas responde a la pregunta por lo que los seres humanos pueden hacer y ser en coherencia con un sentido de dignidad y florecimiento humano. Para Nussbaum (2012) el enfoque de las capacidades se define como una aproximación particular a la evaluación de la calidad de vida y a la teorización sobre la justicia social básica. La autora afirma que para determinar el grado de bienestar de una persona la pregunta central que este enfoque pretende responder es: ¿qué es capaz de hacer y de ser cada individuo digna y libremente? Así, lo importante para evaluar el bienestar de un sujeto determinado no es lo que tiene sino lo que consigue realizar con lo que tiene.

Las capacidades tienen tres formas de comprenderse: una innata, otra interna y otra combinada. Estas tipologías del concepto de capacidad están interconectadas e interdependientes. 1) El nivel innato presenta las condiciones genéticas y biológicas con las que nace la persona. 2) El nivel interno son el conjunto de capacidades que se transforman a través de la educación (conocimientos, habilidades, actitudes y prácticas). Tanto el nivel innato como interno de las capacidades son comprendidos como funcionamientos humanos. 3) El nivel de las capacidades combinadas -comprendidas como funcionamientos institucionales- son las oportunidades y condiciones que se propician para un desarrollo de capacidades internas e innatas idóneas. El último nivel de las capacidades está vinculado con las instituciones y sus estructuras. 
La escuela, desde el enfoque de capacidades, es comprendida como un bien. De acuerdo con Sen (2010), los bienes no son estrictamente económicos; presentan una serie de características que los hacen pertenecientes a una categoría mucho más amplia, y necesarios para los seres humanos. Una buena educación puede constituirse como un bien social por el impacto que causa en quienes tienen acceso a ella. El aspecto valorativo no recae propiamente sobre el bien sino en la medida en que el bien -en este caso, la educación- permita un óptimo funcionamiento de los seres humanos, quienes libremente acceden a él dado que provee y potencia conocimientos y prácticas de sostenibilidad de la democracia, de desarrollo de un proyecto de vida y de acceso al mundo productivo.

Transformar imaginarios sociales hace referencia a realizar cambios importantes en las estructuraciones simbólicas y en cómo los seres humanos representamos lo real. Un imaginario social, según Taylor (2006), determina las imágenes, las ideas y las concepciones comunes que comparte un colectivo y por tanto constituye el telón de fondo desde el cual interpreta sus acciones y modos específicos de organización e interacción. Este proceso implica tener en cuenta tres elementos:

- Teoría implícita: son los conocimientos, principios, creencias y determinantes que fundamentan la acción y que están ocultos o implícitos. Si un sujeto tiene un conocimiento de la norma, mayores elementos tendrá para hacer valer sus derechos. Cuando pensamos en transformar el imaginario estamos pensando en identificar la teoría implícita para ponerla en cuestionamiento.

- Expectativas sociales: surgen del proceso de mostrar a la luz la teoría implícita. Cuando una teoría implícita se torna explícita, aparece una expectativa social de quienes conviven en una comunidad. Este concepto se puede entender también como la forma en que esperamos en que otra persona actúe si ambos viven regidos por un manual de convivencia o si ambos viven en un mismo hogar. Vemos entonces que es cotidiana, que es posible comprenderla, que es un asunto que hace que esperemos algo del comportamiento de los otros y que tiene un diálogo permanente con la teoría implícita.

- Práctica social: las podemos entender como las acciones de los seres humanos en comunidad. Estas acciones las podemos percibir. La actitud, que la entendemos como una disposición, promueve las prácticas: si hay una actitud positiva hacia la paz, posiblemente tenderemos de una mejor manera a realizar acciones que contribuyan a construir paz. 
La pedagogía para la humanización, por naturaleza relacional, se fundamenta, en primer lugar, en los planteamientos de las éticas del cuidado, las cuales hacen énfasis en que todos los seres humanos tienden por su naturaleza a reconocer en los otros seres vivos su existencia y hacer lo que sea necesario para proteger, cuidar y preservar su vida. Las éticas del cuidado no se preocupan tanto por los niveles racionales de toma de decisiones o de vinculación con un acuerdo normativo, los cuales bien podrían ser categorizados en una ética kantiana; tampoco, por la construcción de una virtud cívica del buen ciudadano que configura una vida buena en la polis; son, a lo sumo, éticas de las relaciones cotidianas humanas, construidas a partir del sentimiento natural de responsabilidad y cuidado por el otro.

Noddings (2009) enfatiza que la ética del cuidado es esencialmente relacional y su propósito fundamental es comprender la relación de diálogo, apertura y solidaridad que se produce en la interacción humana de mutuo reconocimiento y de escucha. Bajo este presupuesto, la escuela promueve en la comunidad educativa actitudes de cuidado y de solidaridad cuando enseña sobre el valor de la vida y sobre los principales mecanismos que tienen los seres humanos para protegerla y dignificarla.

Para Nussbaum (2014), las relaciones dadas por las éticas del cuidado son relaciones de mutuo reconocimiento humano y de compasión. En sus palabras: “La compasión es una emoción dolorosa orientada hacia el sufrimiento grave de otra criatura o criaturas" (2014, p. 175). Además de ello, la compasión se compone de tres juicios, los cuales implican una combinación o una predicción, que no pasan por proposiciones formuladas lingüísticamente: (i) Pensamiento de gravedad: “cuando experimenta compasión, la persona piensa que está sufriendo algo importante. Esa es una valoración que se realiza, y que debería realizarse normalmente desde el punto de vista de un espectador o evaluador externo, que es el de la persona que experimenta la emoción" (2014, p. 176); (ii) Pensamiento de la no culpabilidad: “Normalmente no sentimos compasión si pensamos que el problema o la dificultad que padece la persona ha sido escogido por ella misma o se lo ha autoinflingido" (2014, p. 176); (iii) Pensamiento eudemónico: "un juicio o un pensamiento que sitúa a la persona o personas que sufren entre las partes importantes de la vida de la persona que siente la emoción compasiva" (2014, p. 178).

En tercer lugar, las pedagogías de la humanización están fundamentadas en una ética del reconocimiento, en la cual, según Honneth (1997) siguiendo a Mead (1973), el yo no es más que el estímulo que se produce en sí mismo cuando observa cómo reacciona el otro por un comportamiento mío. Se infiere, entonces, que el reconocimiento surge en 
el proceso de interacción con el otro, y en tanto el ente humano se concibe como objeto social de conocimiento.

Para Honneth (1997) el reconocimiento está dado en tres dimensiones: filial, ley y logro social. En primer lugar, el reconocimiento filial está articulado con el apego, la confianza básica, la lealtad y el amor. En segundo lugar, el vínculo del reconocimiento legal se comprende en la relación con el Estado y la práctica de exigencia de los derechos: Tengo derecho a tener derechos, Tengo derecho a pertenecer en una comunidad política. En tercer lugar, el vínculo del reconocimiento como logro social implica trabajar desde la escuela a partir de comprender la diversidad de lo humano y de las relaciones en la cohesión social. Finalmente, Honneth (1997) establece que las experiencias sociales de negación de reconocimiento pueden constituir negativamente en la subjetividad de los individuos, y tienen repercusión en las estructuras normativas políticas, morales y jurídicas.

Como puede observarse en la Figura 2, la propuesta pedagógica Humanizarte tiene como objetivos el desarrollo de capacidades (Nussbaum, 2012), la transformación de imaginarios (Taylor, 2006) y la implementación de pedagogías para la humanización, a través de los siguientes énfasis: anamnético-histórico, estético-expresivo, lingüístico-simbólico y ético-político. El fundamento teórico se constituye en cuatro principios filosóficos (reciprocidad, interdependencia, filialidad y pluralidad), los cuales, articulados con el enfoque de capacidades (Nussbaum, 2012) y con la concepción de cultura como entramado de sentidos (Geertz, 1987), cimientan el Taller de Artesanos por la Paz (inspirado en el texto de Sennett, 2009), compuesto de las estrategias pedagógicas Deliberarte, Reflexionarte, Fraternizarte e Implicarte -cada una se corresponde con un principio filosófico distinto-y de una estrategia transversal denominada Transformarte, pensada para el ejercicio transformador de la práctica pedagógica a partir de la reflexión intencionada. Las estrategias se diseñan en el aula con base en un sentido específico proporcionado por una ruta pedagógica, para el desarrollo de conocimientos, habilidades, actitudes y prácticas (políticas, ético-morales, filiales y plurales) según el ciclo de vida (preescolar, básica o media). La ruta pedagógica contiene los siguientes pasos, cada uno con niveles específicos: acogimiento, reconocimiento de los saberes, ampliación de la mirada, desplazamientos comprensivos, proposición de nuevos lenguajes para la paz, apropiación de las prácticas y reconfiguración del proceso. 


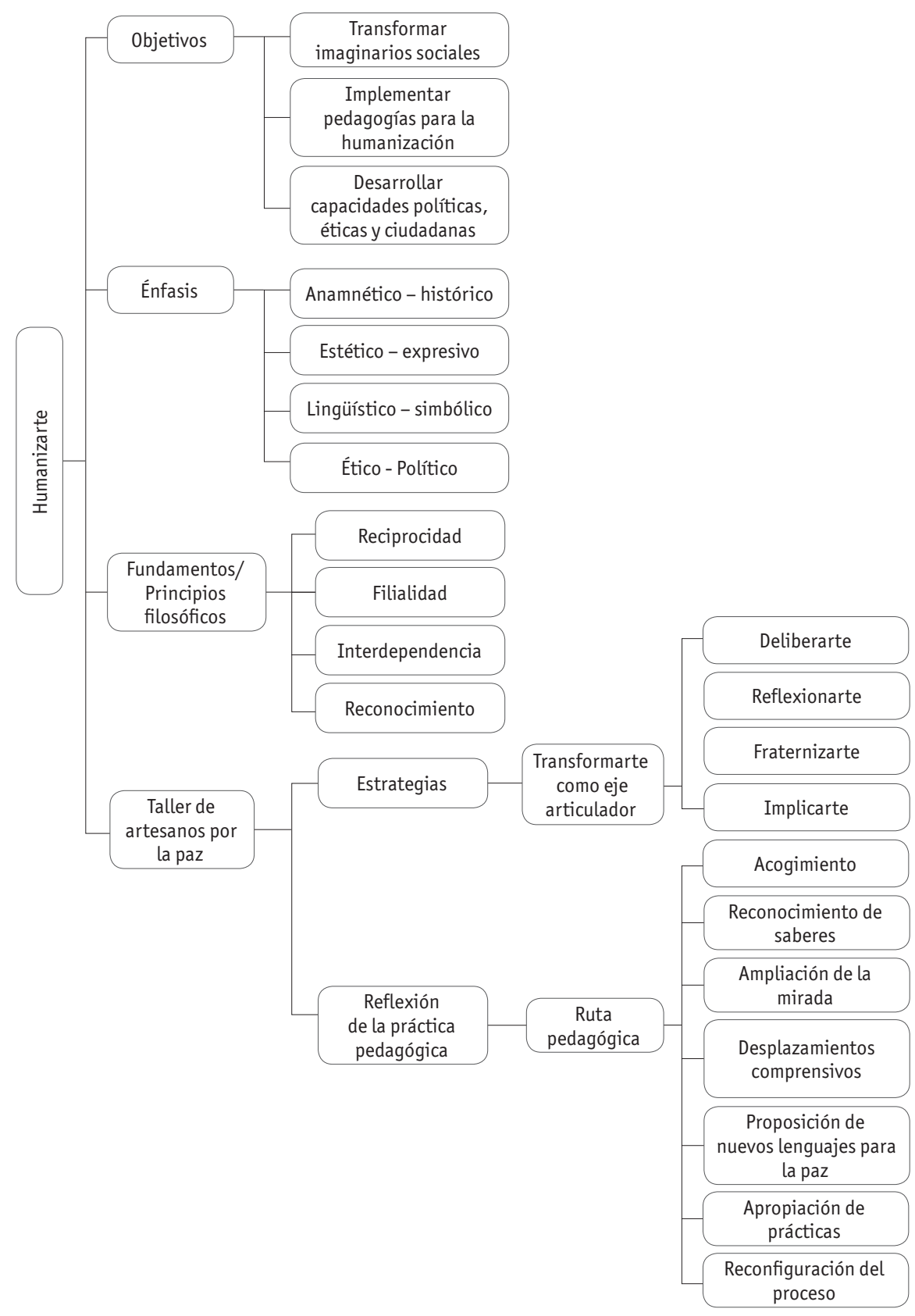

\section{Figura 2. Estructura conceptual de Humanizarte}

Fuente: elaboración propia. 


\section{A manera de discusión y de conclusión}

A partir de los hallazgos de esta investigación es posible deducir que todo proceso de construcción de paz está intrínsecamente relacionado con la constitución de un sujeto político y con el fortalecimiento del ejercicio ciudadano de niñas y jóvenes. Este planteamiento está en coherencia con los planteamientos de Webel (2007), específicamente cuando afirma que los estudios de la paz se han focalizado más en las fronteras, más en las márgenes que en el centro mismo de las disciplinas. A su vez, se concibe la paz como un asunto dialéctico, en que la participación (activa, colectiva e individual) es trascendental, lo cual permite establecer que solo de esta manera es como pueden pensarse los vínculos entre la construcción de paz, las virtudes públicas para la paz y el ejercicio ciudadano desde los derechos.

Con la formación ética y moral de la ciudadanía se brindan elementos para repensar la paz no solo como un derecho y un deber, sino también como una demanda ética y moral que exige la creación de condiciones para que los seres humanos en sus diversas distinciones de lo real puedan convivir sin necesidad de arrasarse unos con otros. Este planteamiento guarda relación con lo dicho por Gur-Ze'ev (2010) cuando estatuye que la paz significa aprender a vivir en conflicto de una manera constructiva; no necesariamente la ausencia de conflicto armado o de guerra.

Esta concepción de paz es similar al concepto de paz positiva acuñado por Galtung (1985), según el cual se define la forma de interdependencia y cooperación entre persones en escenarios de justicia y de no violencia. Otras maneras de concebir el conflicto como oportunidad es a través de un cambio positivo -en función de las habilidades para el manejo de los conflictos y para la construcción de relaciones respetuosas con el otro-; del reconocimiento de las distintas formas de violencia (individual, social y política) y la fascinación por ella -análisis de las experiencias individuales y colectivas de la violencia en el presente y el futuro-; del análisis del impacto, causas y efectos de la guerra -buscar mecanismos contrarios y alternativas a la guerra, en niveles individuales, sociales e internacionales-; del desarrollo de visiones de paz y de vida comunitaria y las maneras de trasladar estas visiones hacia la acción práctica (Jäger, 2014, p. 5). Estas afirmaciones permiten inferir que la formación política, ética y ciudadana debe enfocarse a desarrollar en niñas, niños y adolescentes sus capacidades y posibilidades para ser dignos por sí mismos, respetuosos y cuidadosos con las otras formas de vida humanas y no humanas y profundamente responsables de la construcción de un país más democrático, pacífico, 
justo e incluyente. Adicionalmente a la transformación de imaginarios sociales sobre la paz y las formas no violentas de resolución de los conflictos.

La formación política, ética y ciudadana para la construcción de paz afecta los modos de intervención pedagógica que maestras y maestros llevan a cabo en sus aulas y los diversos escenarios de las instituciones educativas, de manera tal que la intencionalidad formativa de construir paz y convivencia permee las prácticas pedagógicas a fin de que sean afectadas y transformadas hacia la concreción de escenarios más democráticos, participativos, incluyentes y altamente cooperados en la construcción de saberes. Este hallazgo guarda relación con el planteamiento de MacGregor (2014), específicamente en la enunciación según la cual todo educador en el marco de la paz debe considerar los principios filosóficos sobre los cuales ejerce la práctica pedagógica, lo cual lo lleva a reflexionarla desde el impacto que esta puede llegar a tener en la transformación de la cultura.

La consolidación de actitudes favorables a la paz y la convivencia están definidas en gran medida por el desarrollo de capacidades políticas, éticas y ciudadanas que los seres humanos potencian cuando están enfrentados a tener que convivir con otros seres que pertenecen a su misma comunidad política de referencia. Esta idea está relacionada con la propuesta de Chaux y Velásquez (2014), quienes proponen la categoría de competencias ciudadanas para esta finalidad transformadora hacia la paz, entendiendo que las competencias permiten articular la formación académica y de conocimiento con el desarrollo integral comprehensivo, el cual abarca elementos culturales y sociales para el desarrollo de habilidades que permitan la resolución de problemas en contextos cambiantes. Esta intención transformadora de la sociedad y la cultura a través de las competencias ciudadanas (las cuales involucran la participación, la convivencia pacífica y el pluralismo) puede ser desarrollada en tanto la escuela es una institución social legítima en el contexto colombiano. No obstante, lograr estos propósitos es tarea de la comunidad educativa en general, pues de lo contrario quedaría reducida a un trabajo de aula de clase y la formación política para la paz no se agota en dichos escenarios. También están implicadas iniciativas sociales y comunitarias, como lo afirman Rojas (2014) y Wirpsa, Rothschild y Garzón (2014).

En suma, la paz es una construcción social que cada vez más exige la corresponsabilidad de todos los ciudadanos vinculados a una comunidad política. Es una conquista por ser alcanzada, que implica a distintos actores, sectores e instituciones. De manera particular, compromete al sistema educativo nacional y con él la escuela y a sus maestros, para 
que desde su quehacer contribuyan al desarrollo del país desde una perspectiva ética, política y de desarrollo humano integral y sustentable.

La construcción de paz es un proyecto educativo de dignificación y humanización de los ciudadanos, que pretende aportar a la justicia social, al reconocimiento de la diversidad y la pluralidad y a que niñas, niños y jóvenes sean sujetos de su propio desarrollo, interlocutores válidos ante las instancias de poder y sujetos de derechos y de deberes.

Adicionalmente, la construcción de paz es una apuesta política que posiciona a los ciudadanos como seres humanos que constantemente están buscando ser dignos por sí mismos y como ciudadanos que regulan sus acciones de una lectura de los derechos. De ahí, entonces, que una propuesta de construcción de paz desde la escuela necesariamente tenga que estar relacionada con el desarrollo de capacidades políticas, éticas y ciudadanas, así como con la creación de condiciones institucionales que favorezcan el encuentro con el otro bajo condiciones de reconocimiento, dignificación y cuidado. Esto es, una escuela que trabaja arduamente para evitar la humillación, contrarrestar la violencia y promover la denuncia de la violación de derechos. Esto implica reconocer al otro como sujeto de cuidado y de compasión, sujeto de derechos y deberes y sujeto aportante al logro social colectivo.

La construcción de paz está motivada ética y moralmente hacia la configuración de un proyecto de humanidad y, por tanto, está orientada al florecimiento humano. Los variados rostros de humanidad están allí para interpelarse unos a otros y movilizar formas no violentas de convivencia. Cultivar la humanidad, precisamente, está relacionado con estos asuntos. Todos sabemos que continuamente nos enfrentamos unos a otros desde una simple y superficial lectura de nuestros egoísmos, pero el desafío no está allí. El mayor reto está en reconocer rasgos de humanidad que nos llevan a crear condiciones de vivir en la pluralidad. Esto significa explicitar el deseo y disponerse a construir deseos colegiados, comunes y profundamente respetuosos de la particularidad de cada ser humano y de cómo en el ejercicio de su libertad nos impele a ampliar los marcos comprensivos de regulación de la interacción para que estos en su aplicación eviten la humillación, la injusticia y la desigualdad. Esto significa aprender a vivir en la pluralidad. Generar aprendizajes a partir de este principio ético es la condición de toda vida política. 


\section{Referencias}

Adetoro, R. (2015). Effects of learning together, constructive controversy in students' acquisition of knowledge and skills in peace education aspect of social studies. European Researcher, 93 (4), 325-330.

Alvarado, S. (2010). Niños, niñas y jóvenes constructores de paz. En J. Martínez y F. Neira, (comp.), Miradas sobre la reconciliación: reflexiones y experiencias (pp. 141-166). Bogotá: Universidad de Lasalle. Recuperado de http://www.lasalle.edu.co/wps/wcm/connect/ 8263fd4b-f843-4588-aaf6-cfdee13a18a1/catedra-2009.pdf?MOD=AJPERES

Alvarado, S., Botero, P. y 0spina H. (2010). Subjetividades políticas: sus emergencias, tramas y opacidades en el marco de la acción política. Mapeo de 61 experiencias con vinculación de jóvenes en Colombia. Utopia y praxis latinoamericana, (50), 39-55.

Arévalo, J. (2014). Construcción de paz y un nuevo modelo de construcción de Estado: una lectura de los dos primeros acuerdos de La Habana. Revista de Economía Institucional, 16 (30), 131-169.

Bajaj, M. (2015). 'Pedagogies of resistance' and critical peace education praxis. Journal of Peace Education. 12 (2), 154-166. DOI: 10.1080/17400201.2014.991914

Bouvier, V. (ed.) (2014) Colombia: la construcción de la paz en tiempos de guerra. Bogotá: Editorial Universidad del Rosario.

Carter, L., and Shipler, M. (2005). Youth: Protagonist for peace. In P. Tongeren, M. Brenk, M. Hellema, and J. Verhoeven (eds.), People Building Peace II. Successful Stories of Civil Society (pp. 147-157). London: Lynne Rienner.

Chaux, E. (2012). Educación, convivencia y agresión escolar. Bogotá: Taurus.

Chaux, E. y Velásquez, A. (2014). Educación para la paz en Colombia: La promesa de las competencias ciudadanas. En V. Bouvier, ob. cit., pp. 211-224.

Comisión de Sabios (1996). Colombia: al filo de la oportunidad. Bogotá: Tercer Mundo Editores.

Díaz, C. (2011). Pacto regional lasallista por la infancia y la juventud: Un compromiso por la educación, la inclusión y los derechos. Reflexiones y experiencias en la región latinoamericana lasallista. Bogotá: Región Latinoamericana Lasallista (RELAL) y Observatorio educativo Lasallista para los Derechos de la Niñez y la Juventud en América Latina y el Caribe. Recuperado de http://www.relal.org.co/files/Observatorio\%20Educativo\%20Lasallista/ PACTO-REGIONAL-LASALLISTA.pdf

Dubet, F. (2004). ¿Mutaciones institucionales y/o neoliberalismo? En E. Tenti, Gobernabilidad en los sistemas educativos en América Latina (pp. 15-43). Buenos Aires: Instituto internacional de planeamiento de la educación- UNESCO. Recuperado de http://unesdoc. unesco.org/images/0014/001443/144336s.pdf 
Echavarría, C. y Vasco, E. (2013). Las voces de niños y niñas sobre la moral: desafios para la formación ciudadana, ética y política. Bogotá: Universidad de La Salle.

Echavarría, C., Murcia, N., y Castro, L. (2014). Imaginarios sociales de la formación política, ética y ciudadana para la construcción de paz y convivencia en las instituciones educativas del Distrito Lasallista de Bogotá. Bogotá: Distrito Lasallista de Bogotá (Secretaría de Educación) y Universidad de La Salle.

Esquivia, R. y Gerlach, B. (2014). La comunidad local como espacio creativo para la transformación. La perspectiva desde los Montes de María. En V. Bouvier, ob. cit., pp. 377-397.

Fernández, A. (2014). Formación ciudadana: Jóvenes y acción social. Revista Electrónica de Investigación Educativa, 16 (1), 29-42. Recuperado de http://redie.uabc.mx/vol16no1/ contenido-fdezalatorre.html

Gachanga, T., and Mutisya, M. (2015). Interfaith dialogue at peace museums in Kenya. Journal of Peace Education, 12 (3), 277-284. DOI: 10.1080/17400201.2015.1103395

Galtung, J. (1985). Sobre la paz. Barcelona: Fontamara.

Galtung, J. (1990). Cultural violence. Journal of Peace Research, 27 (3), 291-305.

Galvis, C., Moya, L., Garcés, A., Morales, G. y Galeano, Y. (2006). No somos vulnerables. Escuela y niñez en situacion de vulnerabilidad. Bogotá: Instituto para la investigación y el desarrollo pedagógico (IDEP).

Geertz, C. (1987). Interpretación de las culturas. México D.F.: Gedisa.

Gómez, G. (2013). Sistema nacional de convivencia escolar: Una guía para actualizar el manual de convivencia (Ley 1620 de 2013). Bogotá: Magisterio Editorial.

Grau, M. (2013). Recordar para olvidar la desigualdad de género. Revista de derecho público, 1 (31), 1-23. DOI: http://dx.doi.org/10.15425/redepub.31.2013.11

Gur-Ze'ev, I. (2010). Philosophy of peace education in a postmetaphysical era. In S. Gavriel and E. Cairns (eds.), Handbook on peace education (pp. 171-186). New York: Taylor Francis.

Harber, C., and Sakade, N. (2009). Schooling for violence and peace: how does peace education differ from ‘normal' schooling? Journal of Peace Education, 6 (2), 171-187. D0I: http:// dx.doi.org/10.1080/17400200903086599

Henao, H. F. (2014). La iglesia católica colombiana y el trabajo por la paz. En V. Bouvier, ob. cit., pp. 225-248.

Honneth, A. (1997). La lucha por el reconocimiento. Barcelona: Crítica.

Isacson, A. y Rojas, J. (2014). Los orígenes, la evolución y las lecciones del movimiento colombiano por la paz. En V. Bouvier, ob. cit., pp. 39-66.

Jäger, U. (2014). Peace Education and Conflict Transformation. Berlin: Berghof Foundation. Recuperado de http://www.berghof-foundation.org/fileadmin/redaktion/Publications/ Handbook/Articles/jaeger_handbook_e.pdf 
Lewis, E., and Khatari, S. (2015). From clouds of chemical warfare to blue skies of peace: The Tehran Peace Museum, Iran. Journal of Peace Education, 12 (3), 263-276. D0I: 10.1080/17400201.2015.1092710

López de Mesa, C., Carvajal, C., Soto, M., y Urrea, P. (2013). Factores asociados a la convivencia escolar en adolescentes. Educ. Educ., 16 (3), 383-410. Recuperado de http://www.scielo. org.co/pdf/eded/v16n3/v16n3a01.pdf

MacGregor, S. (2014). Prospective philosophical foundations of peace education. In Factis Pax, 8 (2), 150-166. Recuperado de http://www.infactispax.org/volume8dot2/Mcgregor.pdf

Mitchell, C. y Ramírez, S. (2014). Las comunidades locales de paz en Colombia. Una comparación inicial de tres casos. En V. Bouvier, ob. cit., pp. 319-348.

Moncayo, J. (2014). La resistencia civil frente a la guerra en el Magdalena Medio. En V. Bouvier, ob. cit., pp. 349- 354.

Naoufal, N. (2014). Peace and environmental education for climate change: Challenges and practices in Lebanon. Journal of Peace Education, 11 (3), 279-296. D0I: 10.1080/17400201.2014.954359

Noddings, N. (2009). La educación moral. Buenos Aires: Amorrortu.

Nussbaum, M. (2011). Sin fines de lucro: Por qué la democracia necesita de las humanidades. Buenos Aires: Katz.

Nussbaum, M. (2012). Crearcapacidades: Propuesta para el desarrollo humano. Barcelona: Paidós.

Nussbaum, M. (2014). Emociones políticas: ¿Por qué el amor es importante para la justicia? Barcelona: Paidós.

Orozco, L. (2012). Políticas de Educación Superiory su impacto en las instituciones. Bogotá: Universidad de Los Andes.

Page, J. (2014). Peace education. In D. Phillips (ed.), Encyclopedia of Educational Theory and Philosophy (pp. 596-598). California: Sage Publications. DOI: http://dx.doi. org/10.4135/9781483346229

Pinto, L., Torres, D., González, M., y Caicedo, L. (2012). Orientaciones generales para la atención educativa de las poblaciones con discapacidad en el marco del derecho a la educación. Bogotá: Ministerio de Educacion Nacional, Instituto Nacional para Ciegos e Instituto Nacional para Sordos. Recuperado de http://www.colombiaaprende.edu.co/html/micrositios/1752/articles-320765_Pdf_2.pdf

Power, C. (2014). Building a culture of peace to replace the culture of war. Social Alternatives, 33 (4), 47-51.

Programa de las Naciones Unidas para el Desarrollo: PNUD (2003). El conflicto, callejón con salida: Informe Nacional de Desarrollo Humano para Colombia 2003. Bogotá: PNUD. 
Ramírez, C. y Arcila, W. (2013). Violencia, conflicto y agresividad en el escenario escolar. Educ. Educ., 16 (3), 411-429. Recuperado de http://www.scielo.org.co/pdf/eded/v16n3/ v16n3a02

Ramírez, M. C. (2014). Putumayo: negociando paz y visibilidad como sociedad civil en medio del conflicto armado y la guerra contra las drogas. En V. Bouvier, ob. cit., pp. 399-426.

Rettberg, A. (2014). Los empresarios y la paz en Colombia. En V. Bouvier, ob. cit., pp. 249-266.

Rojas, A., Garzón, F., Hoyos, Y., Castillo, E., Guamanga, D., Jojoa, Y., Peña, F., Riascos, D., Solarte, A., Restrepo, E. (2010). Colombia afrodescendiente. Lineamientos curriculares de la cátedra de estudios afrocolombianos. Bogotá: Ministerio de Educación Nacional.

Rojas, C. (2014). Las mujeres y la construcción de la paz en Colombia. En V. Bouvier, ob. cit., pp. 269-291.

Roldán, M. (2014). Cambio de armas. La negociación de alternativas a la violencia en el Oriente Antioqueño. En V. Bouvier, ob. cit., pp. 355-376.

Ross, K. (2015). Quality as critique: Promoting critical reflection among youth in structured encounter programs. Journal of Peace Education, 12 (2), 117-137. D0I: 10.1080/17400201.2014.979400

Salomon, G., and Cairns, E. (2010). Handbook on Peace Education. New York: Psychology Press. Downloaded from http://samples.sainsburysebooks.co.uk/9781136874529_sample_858772.pdf

Sarmiento, A. (2011). Situación de la educación básica, media y superior en Colombia. Una apuesta al cumplimiento del derecho a la educación para niños, niñas y jóvenes. Bogotá: Fundación Corona, Fundación Restrepo Barco, UNICEF, Corporación Región, Fundación Saldarriaga Concha, Universidad de los Andes, CIFE y Universidad del Norte.

Save the Children (2008). Where Peace Begins. Education's Role in Conflict Prevention and Peacebuilding. London: International Save the Children Alliance. Downloaded from www. savethechildren.org.uk/resources/online-library/where-peace-begins

Sen, A. (2010). La idea de la justicia. Bogotá: Taurus.

Sennett, R. (2009). El artesano. Barcelona: Anagrama.

Tabulawa, R. (2013). Teaching and Learning in Context: Why Pedagogical Reforms Fail in Sub-Saharan Africa. Dakar: CODESRIA.

Tamashiro, R., and Furnari, E. (2015). Museums for peace: agents and instruments of peace education. Journal of Peace Education, 12 (3), 223-235. DOI: 10.1080/17400201.2015.1092712

Tanigawa, Y. (2015). The promotion of peace education through guides in peace museums. A case study of the Kyoto Museum for World Peace, Ritsumeikan University. Journal of Peace Education, 12 (3), 247-262. DOI: 10.1080/17400201.2015.1103396

Taylor, C. (2006). Imaginarios sociales modernos. Barcelona: Ediciones Paidós Ibérica. 
Thapa, A., Cohen, J., Guffey, S., and Higgins D'Alessandro. (2013). A Review of School Climate Research. Review of Educational Research, 83 (3), 357-385. DOI: 10.3102/0034654313483907

Torrente, C. y Kanayet, F. (2006). Contribución de las competencias ciudadanas al rompimiento de la violencia en Colombia: Un estudio a nivel nacional con niños, niñas y jóvenes de quinto y noveno grado. Bogotá: Ediciones Uniandes.

Verhagen, F. (2014). Sustainable communities: a lens for envisioning and achieving a community-based culture of social and ecological peace. Journal of Peace Education, 11 (2), 297-316. DOI: 10.1080/17400201.2014.954361

Watanabe, M. (2015). Passing on the history of 'comfort women': The experiences of a women's museum in Japan. Journal of Peace Education, 12 (3), 236-246. D0I: 10.1080/17400201.2015.1092713

Webel, C. (2007). Introducción. Toward a philosophy and metapsychology of peace. In C. Webel and J. Galtung, Handbook of Peace and Conflict Studies (pp. 3-13). New York: Routledge. Downloaded from: http://www.mkgandhi.org/ebks/handbook-of-peace-and-conflict-studies.pdf

Wirpsa, L., Rothschild, D. y Garzón, C. (2014). El poder del bastón. La resistencia indígena y la construcción de la paz en Colombia. En V. Bouvier, ob. cit., pp. 293-315. 
